\title{
Effects of Variable Viscosity and Thermal conductivity on MHD Free Convective Heat and Mass Transfer Flow Past an Inclined Surface with Heat Generation
}

\author{
G. C. Hazarika \\ Department of Mathematics \\ Dibrugarh University \\ Assam, India
}

\author{
Lurinjyoti Gogoi \\ Department of Mathematics Dibrugarh University \\ Assam, India
}

\begin{abstract}
Effects of variable viscosity and thermal conductivity on a steady two dimensional MHD free convective and mass transfer flow past an inclined semi-infinite surface in presence of heat generation, viscous dissipation and Joule dissipation has been studied. Both the fluid viscosity and thermal conductivity are assumed to vary as inverse linear functions of temperature. Using similarity transformations the governing partial differential equations of motion are transformed into ordinary differential equations which are solved numerically together with the boundary conditions by applying shooting method. The numerical results are presented graphically for different values of parameters entering into the problem.
\end{abstract}

\section{Keywords}

Variable viscosity and thermal conductivity, MHD, heat and mass transfer, and shooting method

\section{INTRODUCTION}

The earlier studies on fluid flow problems of different geometries were mostly based on constant physical properties of the fluid. But the work of Herwig and Gerstern [1] proved that these fluid properties may change especially the fluid viscosity. In addition the study of free convection and mass transfer of an electrically conducting fluid under the influence of magnetic field has attracted interest due to its practical applications in different engineering sciences.

MHD free convection heat and mass transfer flow through a porous medium bounded by a vertical surface in presence of hall current was studied by Reddy et al. [2]. Rashidi [3] discussed the free convective heat and mass transfer for MHD fluid flow over a permeable vertical stretching sheet in the presence of the radiation and buoyancy effects. Unsteady MHD free convection heat and mass transfer in a boundary layer flow past a vertical permeable plate with thermal radiation and chemical reaction was analysed by Malapati et al. [4]. Unsteady MHD free convection heat and mass transfer flow past a semi-infinite vertical permeable moving plate with heat absorption, radiation, chemical reaction and Sorret effects was studied by Rao et al.[5]. Pop et al. [6] investigated the influence of variable viscosity on laminar boundary layer flow and heat transfer due to the continuously moving plate. Kabeir et al. [7] deals with the heat transfer with temperature dependent viscosity in a viscous fluid over a stretching sheet in presence of viscous dissipation and internal heat generation. Effects of radiation and variable viscosity on unsteady MHD flow of a rotating fluid from stretching surface in porous medium was analysed by Rashad [8]. MHD free convection heat and mass transfer flow past an inclined surface with heat generation was investigated by Alam et al. [9].
In this paper an attempt has been made to investigate the effects of variable viscosity and thermal conductivity on a steady two dimensional MHD free convective and mass transfer flow past an inclined semi-infinite surface with heat generation, viscous dissipation and Joule dissipation. The fluid viscosity and thermal conductivity are assumed to vary as inverse linear functions of temperature. Using similarity transformations the governing partial differential equations of motion are reduced to ordinary differential equations which are solved numerically for prescribed boundary conditions by applying shooting method.

\section{MATHEMATICAL FORMULATION}

We consider a steady two-dimensional magnetohydrodynamic flow of a viscous incompressible fluid past a semi-infinite inclined plate. Let, $\mathrm{u}$ and $\mathrm{v}$ be the velocity components along $\mathrm{x}$ direction and $\mathrm{y}$ direction respectively where $\mathrm{x}$-axis is considered along the plate and $y$-axis is taken normal to it. A magnetic field of uniform strength $B_{0}$ is acted normal to the direction of the flow.

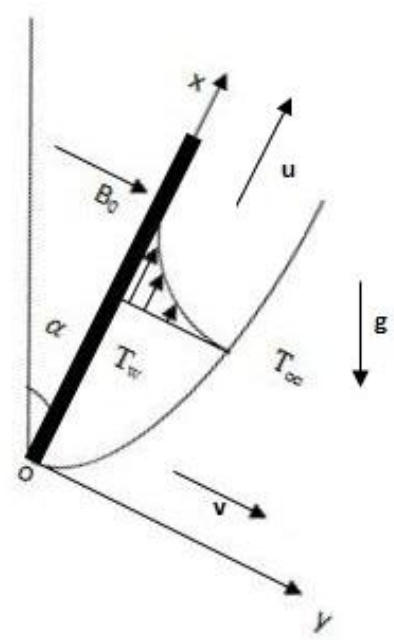

Figure 1: Flow Geometry

The magnetic Reynolds number is assumed as much less than unity so that the induced magnetic field can be neglected in comparison to the applied magnetic field. It is also assumed that all fluid properties are constant except the the fluid viscosity and thermal conductivity which are assumed as inverse linear functions of temperature. Let $\mathrm{T}_{\mathrm{w}}$ and $\mathrm{T}_{\alpha}$ are the temperature of the surface and surrounding fluid respectively where $\mathrm{T}_{\mathrm{w}}>\mathrm{T}_{\alpha}$. Then under the usual Boussinesq's and boundary layer approximations, the governing equations are given by: 
$\frac{\partial u}{\partial x}+\frac{\partial v}{\partial y}=0$

$u \frac{\partial u}{\partial x}+u \frac{\partial u}{\partial y}=\frac{1}{\rho} \frac{\partial \mu}{\partial y} \frac{\delta u}{\delta y}+\frac{1}{\rho} \mu \frac{\partial \mu}{\partial y} \frac{\partial u}{\partial y}+\mathrm{g} \beta\left(\mathrm{T}-\mathrm{T}_{\infty}\right) \cos \alpha+$

$\mathrm{g} \beta^{*}\left(\mathrm{C}-\mathrm{C}_{\infty}\right) \cos \alpha-\frac{\sigma B_{0}{ }^{2}}{\rho} u$

$u \frac{\partial T}{\partial y}+v \frac{\partial T}{\partial y}=\frac{1}{\rho C_{p}} \frac{\partial}{\partial y}\left(K \frac{\partial T}{\partial y}\right)+\frac{Q_{0}}{\rho C_{p}}(\mathrm{~T}-\mathrm{T} \alpha)+\frac{\mu}{\rho C_{p}}\left(\frac{\partial u}{\partial y}\right)^{2}$

$u \frac{\partial C}{\partial x}+v \frac{\partial C}{\partial y}=\frac{\partial}{\partial y}\left(D \frac{\partial C}{\partial y}\right)$

With boundary conditions:

$u=0, v=0, T=T_{w}, C=C_{w}$ at $y=0$

$u=0, T \rightarrow T_{\infty}, C \rightarrow C_{\infty}$ as $\left.y \rightarrow \infty\right\}$

where, $\rho$ is the fluid density, $\mu$ is the coefficient of dynamic viscosity, $\mathrm{T}$ is the fluid temperature, $C_{p}$ is the specific heat at constant pressure, $\sigma$ is the electrical conductivity, $g$ is the acceleration due to gravity, $\beta$ is the coefficient of thermal expansion,

The fluid viscosity is assumed as (Lai and Kulacki [10]),

$$
\left.\begin{array}{c}
\quad \frac{1}{\mu}=\frac{1}{\mu_{\infty}}\left[1+\delta\left(T-T_{\infty}\right)\right] \\
\text { or, } \quad \frac{1}{\mu}=a\left(T-T_{r}\right) \\
\text { where } a=\frac{\delta}{\mu_{\infty}} \text { and } T_{r}=T_{\infty}-\frac{1}{\delta}
\end{array}\right\}
$$

where, $\mu_{\infty}$ is the viscosity at infinity, $a$ and $T_{\infty}$ are constants and their values depend on the reference state and thermal property of the fluid. $T_{r}$ is transformed reference temperature related to viscosity parameter, $\delta$ is a constant based on thermal property of the fluid and $a<0$ for gas, $a>0$ for liquid.

Similarly, the thermal conductivity is taken as,

$$
\begin{aligned}
& \frac{1}{\lambda}=\frac{1}{\lambda_{\infty}}\left[1+\xi\left(T-T_{\infty}\right)\right] \\
& \frac{1}{\lambda}=b\left(T-T_{k}\right) \\
& b=\frac{\xi}{\lambda_{\infty}}, \text { and } T_{k}=T_{\infty}-\frac{1}{\xi}
\end{aligned}
$$

where $b$ and $T_{k}$ are constants and their values depend on the reference state and thermal properties of the fluid.

In order to obtain a similarity solution of the problem we introduce the following non-dimensional variables:

$$
\begin{aligned}
& u=u_{\infty} f^{\prime}(\eta), \eta=y \sqrt{\frac{u_{\infty}}{v_{\infty} x}} \\
& v=\frac{1}{2} \sqrt{\frac{u_{\infty} v_{\infty}}{x}}\left[\eta f^{\prime}(\eta)-f(\eta)\right] \\
& \theta=\frac{T-T_{\infty}}{T_{w}-T_{\infty}}, \phi=\frac{C-C_{\infty}}{C_{w}-C_{\infty}}
\end{aligned}
$$

Now, using equation (6) in equation (2) - (4) we obtain: $\frac{\theta_{r}}{\theta-\theta_{r}} f^{\prime \prime \prime}-\frac{\theta_{r}}{\left(\theta-\theta_{r}\right)^{2}} \theta^{\prime} f^{\prime \prime}-\frac{1}{2} f f^{\prime \prime}+M f^{\prime}-G_{r} \theta \cos \alpha-$ $G_{m} \phi \cos \alpha=0$

$$
\begin{aligned}
& \frac{\theta_{c}}{\theta-\theta_{c}} \theta^{\prime \prime}-\frac{\theta_{c}}{\left(\theta-\theta_{c}\right)^{2}} \theta^{\prime 2}-\frac{1}{2} \operatorname{Pr} Q \theta^{\prime}-\operatorname{Pr} Q \theta+E c \frac{\theta_{r}}{\theta-\theta_{r}} f^{\prime \prime 2}+ \\
& M E c f^{\prime 2}=0
\end{aligned}
$$

$\frac{\theta_{r}}{\theta-\theta_{r}} \phi^{\prime \prime}-\frac{\theta_{r}}{\left(\theta-\theta_{r}\right)^{2}} \theta^{\prime} \phi^{\prime}-\frac{1}{2} S c f \phi^{\prime}=0$

The boundary conditions (5) then turn into:

$f=0, f^{\prime}=0, \theta=0, \phi=0$ at $\eta=0$

$f^{\prime}=0, \theta=0, \phi=0$ as $\eta \rightarrow \infty$

The dimensionless parameters introduced in the above equations are defined as follows:

$\mathrm{M}=\frac{\sigma B_{0}^{2} x}{\rho U_{\infty}}$ is the local magnetic field parameter,

$\mathrm{Gr}=\frac{g \beta\left(T_{w}-\mathrm{T}_{\alpha}\right) x}{U^{2}}$ is the local Grashof number heat transfer,

$\mathrm{Gm}=\frac{g \beta^{*}\left(C_{w}-C_{\infty}\right) x}{U_{\infty}^{2}}$ is the local Grashof number mass transfer,

$\operatorname{Pr}=\frac{v_{\infty} \rho c_{p}}{K}$ is the Prandtl number,

$Q=\frac{Q_{0} x}{\rho c_{p} U_{\infty}}$ is the local heat generation parameter

and $\mathrm{Sc}=\frac{v_{\infty}}{D}$ is the Schmidt number.

\section{RESULTS AND DISCUSSION}

The variations in velocity profile, temperature profile and concentration profile are displayed graphically in figure 2 to figure 13. The graphical representations of velocity profile for various values of parameters are shown in figure 2 to figure 6 . From the figure 2 it is found that the velocity of the fluid first increases near the plate and then gradually decreases. Figure 3 and figure 6 shows that velocity of the fluid decreases as the Hartmann number $\mathrm{M}$ and inclination angle $\alpha$ increases. Due to the increase of Hartmann number $M$ the Lorentz force which is a flow resistive force increases as a result velocity of the fluid decreases. It is seen in the figure 4 and figure 5 that velocity of the fluid increases when Grashof number for heat transfer $\mathrm{Gr}$ and Grashof number for mass transfer $\mathrm{Gm}$ increases. Increase of Grashof number for heat transfer $\mathrm{Gr}$ and Grashof number for mass transfer $\mathrm{Gm}$ means increase of buoyancy force and decrease the viscosity due to which velocity of the fluid increases.

The graphs of temperature profile for the variation of viscosity parameter $\theta_{r}$, thermal conductivity parameter $\theta_{c}$, heat generation parameter $\mathrm{Q}$ and Hartmann number $\mathrm{M}$ are presented in figure 7 to figure 10 . In figure 7 , figure 9 and figure 10 it is observed that temperature of the fluid in the boundary layer increases when viscosity parameter $\theta_{r}$, heat generation parameter $\mathrm{Q}$ and Hartmann number $\mathrm{M}$ increase. It is due to the fact that when viscosity parameter $\theta_{r}$ and Hartmann number $\mathrm{M}$ increase resistance for the flow increases for which heat is generated so temperature of the fluid increases. Due to the increase of thermal conductivity parameter $\theta_{c}$ the temperature of the fluid decrease which is seen in the graph of figure 8 . As the conductivity of the fluid increases, the transfer of heat increase so temperature of the fluid decrease.

The variation of concentration profile for the variation of viscosity parameter $\theta_{r}$, Hartmann number $\mathrm{M}$ and Schmidt number Sc are shown in figure 11 to figure 13. In figure 11 and figure 12 it is seen that the concentration of the fluid increases with the increasing values of viscosity parameter $\theta_{r}$ and Hartmann number $M$. Increasing values of viscosity parameter $\theta_{r}$ and Hartmann number M retard the velocity of the fluid so the concentration of the fluid increases. Figure 13 shows that concentration of the fluid decreases with the increase of Schmidt number Sc. 


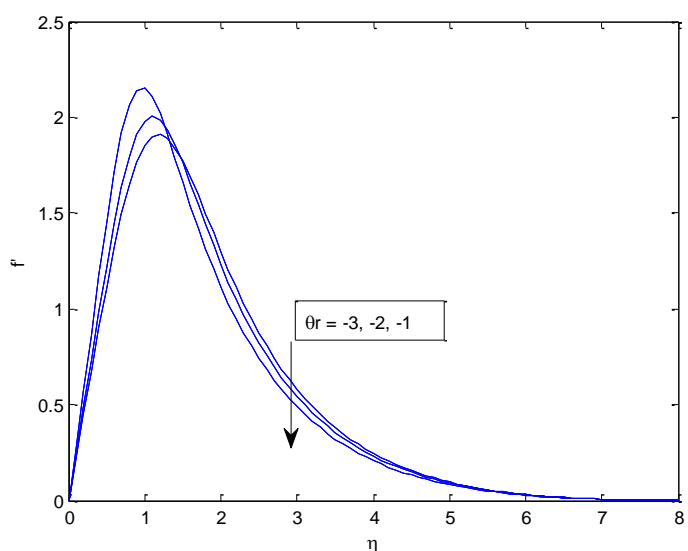

Figure 2: Velocity profile for different $\theta_{r}$

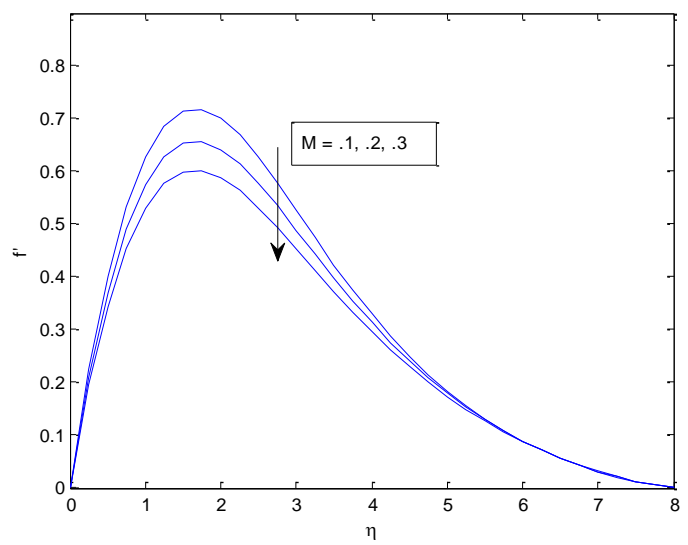

Figure 3: Velocity profile for different $M$

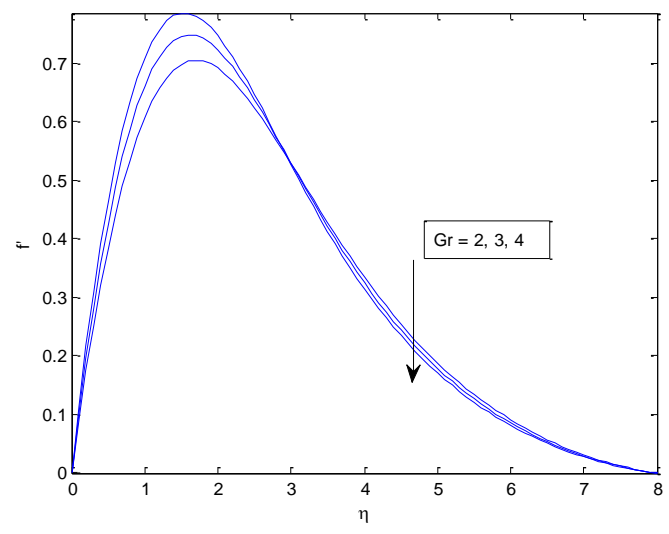

Figure 4: Velocity profile for different $\mathrm{Gr}$

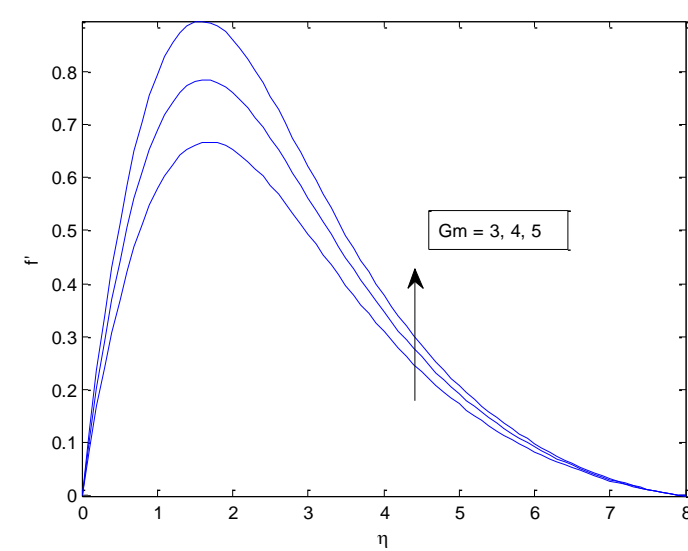

Figure 5: Velocity profile for different $\mathbf{G m}$

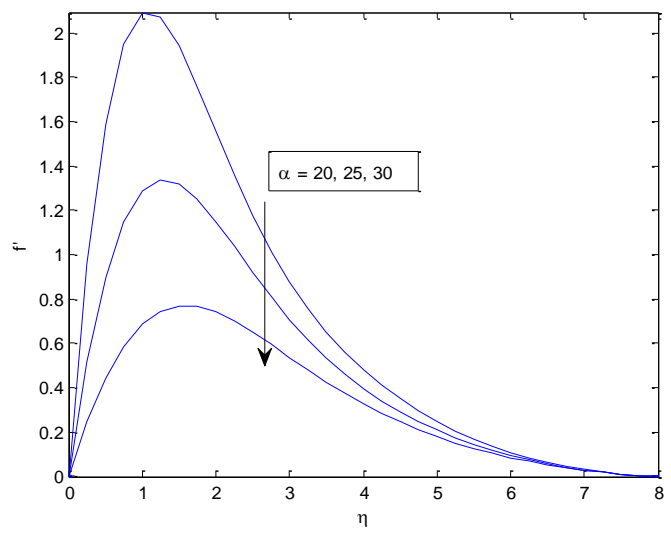

Figure 6: Velocity profile for different $\alpha$

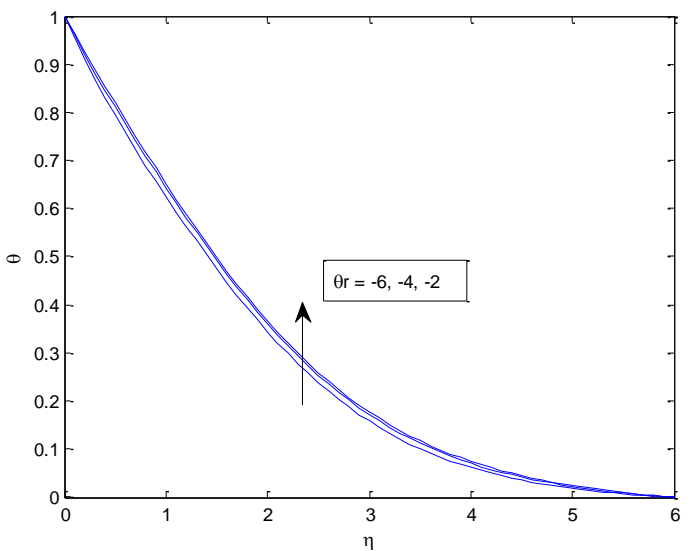

Figure 7: Temperature profile for different $\theta_{r}$ 


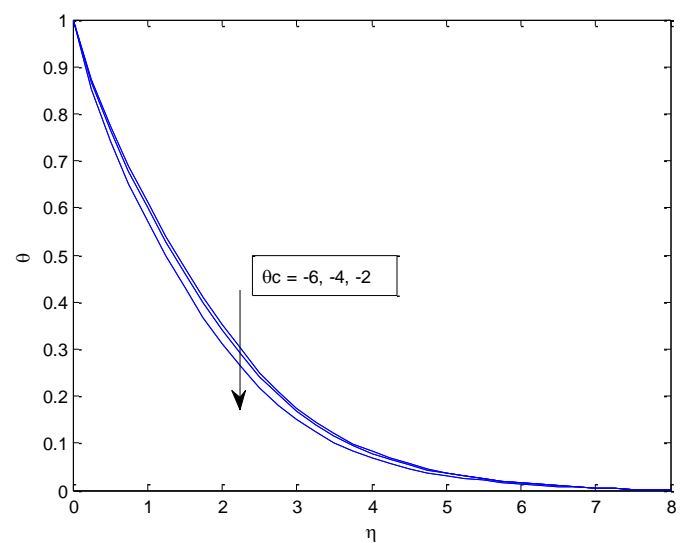

Figure 8: Temperature profile for different $\theta_{c}$

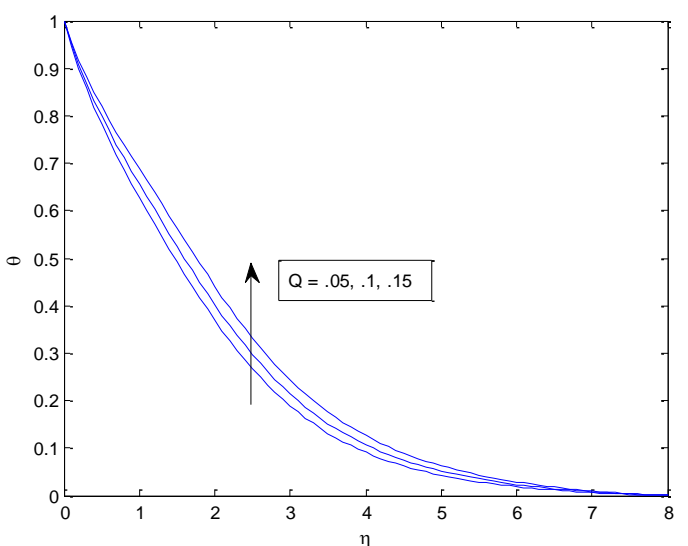

Figure 9: Temperature profile for different $Q$

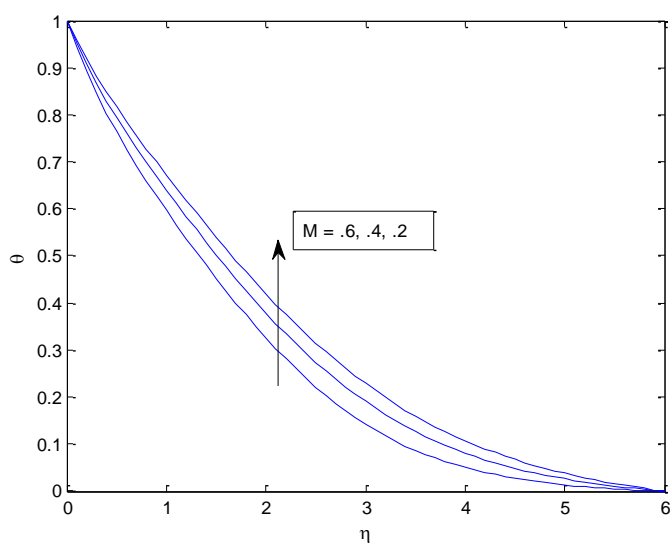

Figure 10: Temperature profile for different $M$

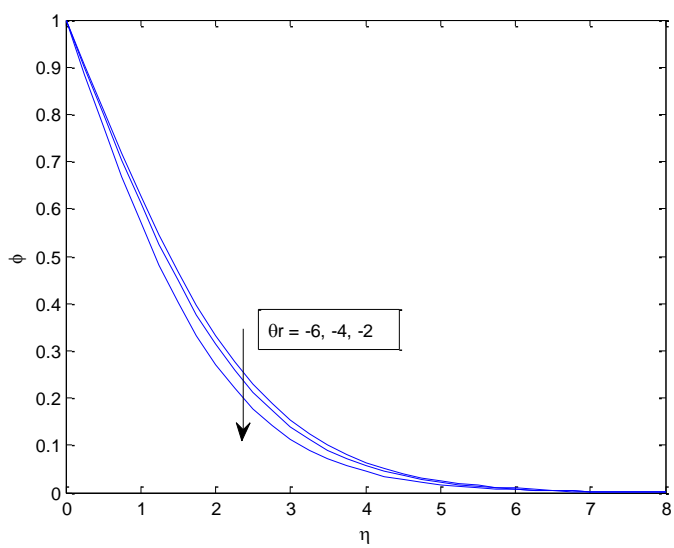

Figure 11: Concentration profile for different $\theta_{r}$

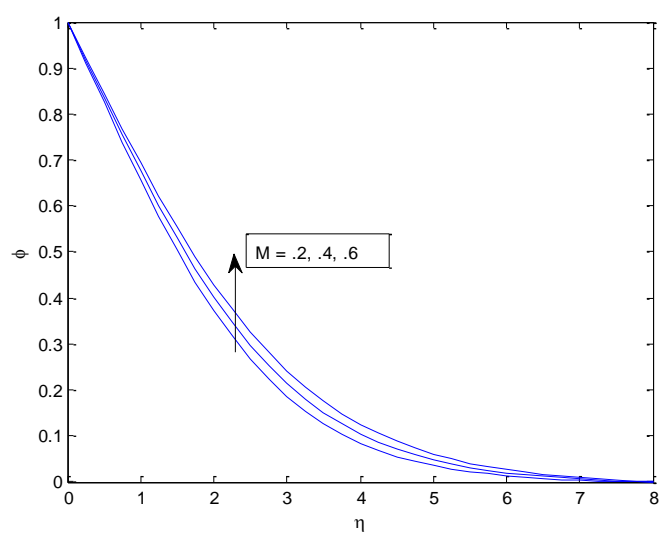

Figure 12: Concentration profile for different $M$

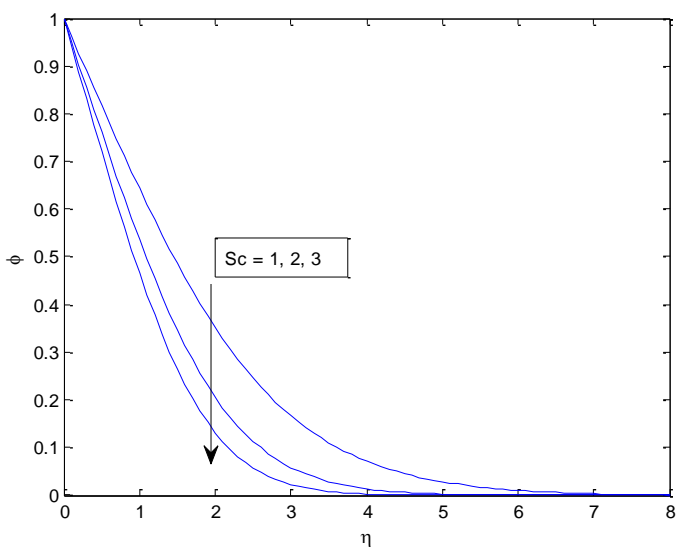

Figure 13: Concentration profile for different Sc

\section{CONCLUSIONS}

Effects of variable viscosity and thermal conductivity on a steady two dimensional MHD free convective and mass transfer flow past an inclined semi-infinite surface in presence of heat generation, viscous and Joule dissipation has been studied. Following conclusions can be drawn from the above study.

i. Temperature increases due to the increase of viscosity parameter $\theta_{r}$ whereas thermal conductivity parameter $\theta_{k}$ decreases the temperature of the fluid. 
ii. Increase of magnetic field intensity decrease the velocity but decrease the temperature of the fluid.

iii. Increasing values of Grashof number enhance the velocity of the fluid.

iv. When Schmidt number increases the concentration of the fluid decreases.

\section{REFERENCES}

[1] H. Herwig, K. Gersten, Warme and Staffubertr, vol. 20, pp. 47,1986 .

[2] T. S. Reddy, O. S. P. Reddy, M. C. Raju and S.V.K Varma, Advances in Applied Science Research, vol. 3, No. 6, 3482-3490. 2012

[3] M. M. Rashidi, B. Rostami, N. Freidoonimehr, S. Abbasbandy, Ain Shams Engineering Journal, vol. 5, 2014, 901-912.
[4] V. Malapati and P. Polarupa, Procedia Engineering, vol.127, 2015, 791-799.

[5] B. M. Rao, G. V. Reddy, M. C. Raju and S.V.K. Varma, Int. J. Eng. Sci and Emerging Tech., vol. 6, No. 2, 2013, 241-257.

[6] I. Pop, R.S.R. Gorla and M. Rashidi, Int. J. Eng. Sci., vol. 30, 1992, 1-6.

[7] S.M.M. EL-Kabeir and R.S.R. Gorla, Int. J. Fluid Mechanics Research, vol. 34, 2007, 42-51.

[8] A.M. Rashad, Journal of the Egyptian Mathematical Society, vol. 22, 2014, 134-142

[9] S. Alam, M.M. Rahman and M.A. Sattar, Thammasat Int. J. Sc. Tech., vol. 11, No. 4, 2006.

[10] F.C. Lai, F.A. Kulacki, Int.J.Heat Mass Transfer, vol. 33, 1990, 1028-1031. 